\title{
Traitement Des Investissements Étrangers Dans Les Législations Nationales: Regard Croisé Sur Les Incitatifs Du Code Burundais Des Investissements De 2008
}

\author{
Anaclet Nzohabonayo \\ Docteur en droit; \\ Enseignant-chercheur et Chef de département Administration à l'Ecole \\ Nationale d'Administration/ Bujumbura-Burundi \\ Professeur visiteur à l'Université Officielle, à l'Université Libre \\ des Grands lacs et à l'Institut Supérieur Pédagogique \\ de Bukavu en République démocratique du Congo
}

\section{Doi:10.19044/esj.2018.v14n16p199 URL:http://dx.doi.org/10.19044/esj.2018.v14n16p199}

\begin{abstract}
This reflection assesses the incentives of the Burundian code of investments of 2008 as for their extent and effectiveness. It shows that the 2008 Burundi Investment Code has created a favorable business climate. It provides for very generous tax and customs incentives that can influence foreign investors in the choice of destination for their investments. However, these incentives have been constantly nibbled following the entry into force of the budget laws of 2016 and 2017 and even a straddle of the general tax code of 2013. From the analysis, it appears that these incentives have lost their substance and that the 2008 code and the Free Zone Act of 2001 have become lapsed instruments that display incentives that have been flawed to the point that some have even been removed. Given the fact that there is a gap between the planned and the actual incentives, there is a need to compile these incentives to identify those that have become archaic and to redefine them to make a new one. The ultimate outcome of this work would be the revision of the investment code of 2008. It is in this respect that the Burundian legislator could reinforce predictability and transparency and keep in the logic of increasing the flow of foreign investments that can generate new sources of financing for development.
\end{abstract}

Keywords: Burundi, investment, fiscal holiday, investment code, Burundi investment authority 


\section{Résumé}

Cette réflexion évalue les incitatifs du code burundais des investissements de 2008 quant à leurs étendue et effectivité. Elle démontre que le code des investissements burundais de 2008 a inauguré un climat favorable aux affaires. Il prévoit des incitatifs fiscaux et douaniers pouvant influencer les investisseurs étrangers dans le choix de destination de leurs investissements. Toutefois, lesdits incitatifs ont subi un grignotage incessant consécutif à l'entrée en vigueur des lois budgétaires de 2016 et 2017 et même un enjambement du code général des impôts de 2013. Nous soutenons que ces incitatifs sont plus figuratifs qu'existants et que le code de 2008 ainsi que la loi de 2001 sur la zone franche sont devenus des instruments caducs déroutants. Eu égard à ce constat de décalage entre les incitations prévues et celles qui sont effectives, il y a la nécessité de faire un travail de compilation de ces incitatifs pour en détecter ceux qui sont devenus archaïques et procéder à leur redéfinition pour en effectuer une nouvelle codification. L'ultime aboutissement de ce travail serait la révision du code des investissements de 2008. C'est à ce titre que le législateur burundais pourrait renforcer la prévisibilité et la transparence et maintenir la logique du code d'accroitre le flux des investissements étrangers pouvant générer de nouvelles sources de financement du développement.

Mots clés : Code des investissements, zone franche, incitatifs, investissements Burundi

\section{Introduction générale}

Eu égard au recul de l'aide au développement et aux conditionnalités des bailleurs de fonds qui imposent des contraintes pour accéder au financement, les pays en développement ont opté pour d'autres mécanismes pouvant générer des sources de financements de leurs projets de société. Une des alternatives a consisté à la mise en place des codes d'investissements comportant des clauses fiscalement et socialement généreuses. Ces dispositions visent à attirer les flux d'investissements étrangers afin de mobiliser des recettes publiques permettant de répondre aux impératifs de relèvement du bien-être des citoyens. Le législateur burundais s'est très tôt inscrit dans ce schéma de stimulation des investissements étrangers en mettant en place des codes d'investissements pour optimiser les rentrées dans les caisses du trésor public (Codes des investissements de 1963, 1967, 1979 et 1987).

Notre préoccupation est d'évaluer les incitatifs du code burundais des investissements de 2008 (Code de 2008) quant à leur étendue et effectivité. Nous postulons que lesdits incitatifs ont subi un grignotage incessant 
consécutif à l'entrée en vigueur des lois budgétaires de 2016 et 2017 et même un enjambement du code général des impôts de 2013. Beaucoup de ces incitatifs ont perdu de leur substance et le code de 2008 ainsi que la loi sur la zone franche sont devenus des instruments caducs contenant des incitatifs que le cadre légal et réglementaire a écorchés au point que certains ont plutôt été supprimés. Eu égard à ce constat de décalage entre les incitatifs prévus et ceux qui sont effectifs, l'étude considère la révision du code actuelle des investissements comme un impératif méritant une attention urgente.

La gestion des incitatifs relève des structures dont les organes se trouvent aux différents échelons de commandement dans la hiérarchie administrative. Pour conduire l'évaluation des incitatifs, l'analyse porte sur la stratégie attractive que prône le code burundais des investissements de 2008 . Nous étudions le dispositif incitatif du code de 2008 pour en dégager la nature et la portée. La réflexion appréhende les fondements de cette philosophie incitative du code (I) sous l'angle de la volonté de rupture avec la dépendance économique (A) et de l'inauguration d'un climat favorable aux affaires (B). L'examen des incitations prévues dans le code (II) est organisé en deux temps. D'une part, les incitatifs sont analysés sous le prisme du régime général (A) et d'autre part sous l'empire de la zone franche (B). Nous clôturons notre analyse par une conclusion.

\section{Philosophie incitative dans le code des investissements burundais}

Nous estimons qu'il est tout à fait légitime de s'interroger sur les motivations du législateur burundais en instituant les multiples avantages douaniers, fiscaux et sociaux en faveur des investisseurs étrangers. Nous scrutons la volonté de rupture avec la dépendance économique (A) ainsi que le souci d'aménager un climat favorable aux affaires (B) comme les principaux déterminants des incitatifs contenus dans le code des investissements.

A. Volonté de rupture avec la dépendance économique

L'élaboration des codes d'investissement dans les pays nouvellement décolonisés s'inscrivait dans la problématique du développement économique qui hantait ces nouveaux États. Le Burundi, à l'instar d'autres anciennes colonies d'Afrique, a hérité d'une économie dont il fallait s'approprier les orientations politiques pour prendre en main son destin économique. Dès lors, les nouveaux dirigeants centraient leurs préoccupations sur la redéfinition d'une politique économique répondant aux principes d'autonomie et d'indépendance. Ils constatent très vite que celle-ci n'est qu'un leurre si elle n'est pas accompagnée de plans de fiabilisation économiques pour poser les bases d'un développement garant d'une rupture avec la dépendance occidentale.

La rupture relationnelle parfois « douloureuse » et encore fraiche dans les esprits des anciens «maitres» compliquait les liens de coopération 
économique. L'orgueil indépendantiste ou souverainiste justifiait par ailleurs le refus, sans succès, de tendre les mains envers ceux-là à qui on a vanté sa maturité à conduire le peuple décolonisé à la prospérité économique. L'éloignement progressif de cette dernière dans plusieurs des anciennes colonies les a contraintes à l'endettement. Celui-ci a hypothéqué leur capacité d'emprunt sur le marché des capitaux. L'aide publique au développement, souvent critiquée quant à son efficacité, et qui dans le temps a constitué une bouée de sauvetage, s'est raréfiée et est devenue beaucoup conditionnée.

Pour empêcher, sinon retarder, l'étranglement économique, le Burundi à l'instar des autres pays décolonisés, a adopté une politique de promotion et de protection des investissements étrangers (Laviec 2015). Pour incarner cette volonté politique, la philosophie incitative des investissements a guidé la rédaction des diverses générations de codes d'investissement. Il s'agit d'inaugurer un climat favorable aux investissements dont les traits caractéristiques sont les incitatifs douaniers et fiscaux accordés aux investisseurs et investissements étrangers. Au-delà de ses avantages pécuniaires, le code des investissements des assouplissements dans l'application de la législation du travail. Cette politique incitative est complémentée par une protection procédurale et substantielle définissant le traitement à accorder aux investissements étrangers et les garanties de recouvrer leurs droits en cas de non-respect des engagements par les pays d'accueil.

Dés 1963, le Burundi se dote de son premier code des investissements (Loi du 6 août 1963). Après quatre ans d'application, un deuxième code a vu le jour en 1967 (Décret-loi no 1/82 du 25 août 1967). Celui-là est resté en vigueur pendant 12 ans et en 1979, un troisième code a été mis en application (Décret-loi no 1/8 du 4 avril 1979). En 1987, le Burundi a institué un quatrième code d'investissements (Loi no 1/005 du 14 janvier 1987) qui a été abrogé par celui de 2008 actuellement en vigueur.

Cette succession de génération de codes reflète le souci d'ajustement constat au contexte fluctuant des investissements pour tenir en considération les nouveaux développements. Mais, elle matérialise aussi la volonté du législateur de rompre avec l'hostilité et la méfiance envers les investisseurs étrangers qui ont caractérisé les premières années des indépendances des colonies occidentales.

B. L'inauguration d'un climat favorable aux investissements

Plusieurs éléments permettent de caractériser un climat favorable aux affaires. Dans le domaine spécifique des investissements, le code de 2008 comporte des clauses qui instituent les mécanismes alternatifs de règlement des différends tout en libéralisant la saisine des juridictions en cas de différends opposant les investisseurs étrangers au pays d'accueil. Le climat favorable est aussi analysé sous l'angle de la liberté d'entreprendre et des 
facilités instaurées dans l'accomplissement des formalités pour la création des entreprises.

B.1. Libéralisation de la saisine des juridictions en cas de différend entre État et investisseur

Cette libéralisation se manifeste par le droit reconnu aux investisseurs étrangers de saisir les juridictions locales et même étrangères en cas de survenance de différends qui les opposent avec les pays d'accueil. Du reste, le code de 2008 prévoit le recours au règlement amiable des différends.

\section{Recours aux juridictions locales}

Dans le code des investissements de 2008, le Burundi ne renonce pas au recours à ses juridictions nationales. Ses rédacteurs ont préconisé que le règlement des différends qui ne sont pas réglés à l'amiable se réalise conformément aux lois et règlements de fond et de procédure en vigueur au Burundi(Code des investissements de 2008, art 17.1). Dans les faits, ce recours reste hypothétique et par conséquent plus théorique que pratique.

Le code lui-même prévoit l'option de saisine des juridictions arbitrales internationales (Art 17.1). L'arbitrage international offre un forum de règlement qui emporte l'adhésion des investisseurs étrangers par son impartialité et neutralité «supposée ou avérée». Ce faisant, le législateur burundais s'inscrit dans l'optique des rédacteurs de la Convention CIRDI (Centre international pour le règlement des différends en matière d'investissement) qui ont écarté les tribunaux locaux du pays d'accueil pour des préoccupations d'impartialité. À tort ou à raison, les investisseurs étrangers redoutent le manque d'indépendance des juridictions locales pour censurer les comportements des États auteurs des actes à l'origine des violations de leurs droits (CNUCED, 2010).

Nous estimons que dans cette disposition sur le règlement des différends, le gouvernement burundais a manqué l'opportunité de réaffirmer sa souveraineté et de permettre à ses institutions de redresser la situation. Il aurait été plus rationnel de prévoir que le conflit est soumis aux juridictions locales pour une période n'excédant pas 12 mois. L'option de saisine des juridictions arbitrales internationales n'étant envisageable que si l'investisseur n'est pas satisfait de la décision rendue par les juridictions nationales.

\section{Recours au règlement amiable}

En plus des mécanismes contentieux, le code introduit le règlement des différends par voie amiable (Art 17.1). Celle-ci constitue un mode de règlement extrajudiciaire des différends. Autrement, les parties conviennent, de commun accord, d'éviter le recours aux juridictions arbitrales ou étatiques. Elles choisissent entre la négociation directe, la conciliation, la médiation, la constatation et l'évaluation des faits (CNUCED, 2010). 
L'État et l'investisseur étranger, par voie de négociation directe, privilégient les chances de continuité de l'exploitation pour préserver les intérêts mutuels. Elles évitent des procédures contentieuses dont l'issue est la rupture des relations d'affaires qui entraine inéluctablement la cessation des activités économiques. Or, en matière d'affaires, une séparation contentieuse n'est jamais souhaitable. Elle écorne l'image du pays d'accueil envers les investisseurs étrangers et compromet les chances d'en attirer des nouveaux.

Dans la négociation directe, les parties en conflit solutionnent leur litige sans l'intermédiation d'une tierce personne (CNUCED, 2010). Elles mènent entre elles «des contacts personnels visant à se faire part mutuellement de leurs souhaits et de leurs propositions. Elles n'impliquent pas, normalement, l'intervention de tiers à des fins d'assistance ou de facilitation» (CNUCED, 2010 p.14). Les négociations directes sont préférables. Mais, leur aboutissement à une solution durable est sujet à plusieurs variables. Il s'agit notamment, mais non exclusivement, de la volonté des parties à coopérer, de leur capacité à communiquer ainsi que de l'engagement ferme à appliquer la solution négociée.

Parfois les positions des parties en conflit sont radicales au point que le rapprochement des positions rend inévitable le recours à une tierce personne. Dans ce cas, les parties perdent une certaine maitrise du conflit, car l'intervention d'une tierce personne devient un impératif pour résoudre le litige. Les parties désignent alors soit un conciliateur ou un médiateur.

Ces intermédiaires s'évertuent à encourager les parties à solutionner leur litige et à améliorer la communication entre elles. S'appuyant sur les conseils des intermédiaires (conciliateurs ou médiateur), les parties cherchent à régler les problèmes de fond que pose leur différend (CNUCED, 2010). La procédure de règlement à l'amiable, en matière des litiges d'investissement, débouche sur un accord ou tout le moins des recommandations écrites.

Dans le cadre du CIRDI, les procédures de conciliation organisées aboutissent à une décision n'ayant toutefois pas de caractère contraignant à l'égard des parties (CNUCED, 2010). En résumé, le but de la conciliation n'est pas d'améliorer les rapports entre les parties; c'est de trouver une solution concrète à un différend (CNUCED, 2010).

\section{Saisine des tribunaux arbitraux internationaux}

Aux termes des garanties offertes, le code consacre le droit de saisine des juridictions étrangères lorsqu'un investisseur étranger est lésé par un mauvais traitement du gouvernement burundais. Ainsi prévoit-il que " $[L] e$ règlement des différends peut-être réalisé, au choix de l'investisseur, par un arbitrage institutionnel interne ou par un arbitrage international. Lorsqu'il est fait recours à l'arbitrage international, celui-ci se conformera aux règles d'arbitrage du Centre International pour le Règlement des Litiges relatifs aux 
investissements en vigueur au moment de la réalisation des investissements auxquels le différend est lié » (Art 17 (2)).

Cette soumission aux tribunaux arbitraux internationaux constitue une garantie fondamentale dans la protection des investissements étrangers. Sans hésitation, le Burundi assume son choix pour la promotion de développement économique en ouvrant l'option de saisine des juridictions arbitrales étrangères au profit des investisseurs lésés. En contrepartie, il espère une contribution en termes de transfert de technologie, de création d'emplois et de stimulation de la compétitivité des opérateurs économiques locaux.

En acceptant cette compétence juridictionnelle du CIRDI dans les conflits d'investissement, ce code coupe court avec les risques d'impartialité et d'indépendance qui entachent les juridictions nationales statuant dans les affaires où l'État intervient en tant que partie. En renonçant à la justice étatique dont il est le «parrain», le Burundi a manifesté son attachement à l'accueil bienveillant des investisseurs étrangers. La préférence de ceux-ci a toujours porté sur le CIRDI plutôt que les juridictions locales (Houben, 2016).

\section{Garantie d'entreprise et de droit de propriété}

L'autre trait marquant de la législation burundaise sur les investissements concerne la liberté d'entreprendre que le code garantit aux investisseurs étrangers. Au titre de cette liberté, tout investisseur, personne physique ou morale, a le droit de se livrer à l'activité commerciale de son choix et de s'établir où bon lui semble sur le territoire burundais. Relativement à l'activité exercée, la loi y apporte une limite en soumettant l'investissement dans certains secteurs à l'autorisation par des lois particulières. Toujours au chapitre des garanties, des dispositions permettant d'opérer l'investissement accordent des facilités migratoires aux travailleurs expatriés (Code des investissements de 2008).

La dimension économique de l'investissement postule l'existence d'un droit de propriété énergiquement protégé. Le code de 2008 s'en préoccupe et s'interdit toute discrimination en la matière que ce soit pour la protection ou pour l'acquisition du droit de propriété (Art 9 et 10.1). Cet engagement constitue un signal fort de renonciation sans équivoque de mettre en péril le droit de propriété que ce soit au moyen des nationalisations, des expropriations ou des mesures de portée équivalente. Dans cette législation, 1'expropriation s'apparente à une exception qui n'intervient que pour cause d'utilité publique et qui doit être menée de façon régulière moyennant une indemnisation juste, prompte et préalable (Art 13).

Ce droit de propriété rime avec celui de transférer les capitaux ainsi que les revenus professionnels et les rémunérations du capital. Le code autorise aux investisseurs étrangers de les transférer aux destinations de leur choix à condition de s'acquitter des impôts auxquels ils sont assujettis 
(Art 11). Tout compte fait, ces facilités sont disponibles après paiement des impôts. Elles peuvent subir des restrictions qu'imposent des lois en matière de change (Art 11 et 12).

En dépit de l'exigüité foncière au Burundi, le code surprend par les facilités d'installation qu'il aménage au profit des investissements étrangers. Il prévoit la possibilité d'acquisition souple d'espace physique pour leurs investissements, de déclencher, à leur bénéfice, la procédure d'expropriation pour cause d'utilité publique (Art 10).

\section{Assouplissement des formalités de création des entreprises}

Les concepteurs du code de 2008 se sont préoccupés de mettre en place un cadre institutionnel d'encadrement des investissements au Burundi. L'article 18 du code consacre la création de l'Agence de promotion des investissements (API). Celle-ci offre aux investisseurs étrangers l'opportunité d'accomplir toutes leurs formalités administratives et de recevoir les permis exigés ainsi que les services dont ils ont besoin en un point unique appelé "guichet unique» ou «one-stop shop » en anglais (Agence égyptienne de promotion des investissements).

Cet aménagement qui concentre l'exécution de toutes les formalités en un seul endroit résout la question de lenteur administrative qui retardait les décisions et décourageait les investisseurs étrangers. Il permet de réaliser une économie de temps et de ressources. L'API est actuellement en train de concevoir des statuts types sociétés financières et coopératives tout en réactualisant les anciens statuts.

En résumant les fonctions des API, on pourrait dire qu'elles sont «chargées de diffuser une image favorable du pays, de démarcher les industriels et de les informer sur les opportunités d'affaires existantes, de les accueillir et de les conseiller lors de leur implantation effective» (Hatem, 1993, p. 10).

Bien qu'il n'y ait pas de répartition uniforme, les activités qui relèvent de ces fonctions se ramènent à 4 catégories (Kelly, 2004 p. 1). Celles-ci sont les suivantes : soigner l'image du pays pour en créer une perception positive comme destination idéale de l'investissement international; stimuler des investissements étrangers ; fournir des services avant et après investissement ; promouvoir les politiques d'assainissement du climat d'investissement.

En plus des garanties procédurales et d'une protection substantielle accrue, le code confère des avantages douaniers et fiscaux aux entreprises dans le cadre du régime de droit commun et du régime de zone franche (Art 4). Plus encore, les investisseurs étrangers pourraient se prévaloir de l'octroi des avantages plus favorables prévus dans les Conventions ratifiées par le Burundi (Code de 2008, Art 5). 


\section{Regard sur les incitations du code}

Les avantages fiscaux, douaniers et sociaux dont bénéficie l'investisseur étranger sont définis dans le code de 2008 ; celui-là qui incorpore en son sein les dispositions de la loi no 1/015 du 31 juillet 2001 portant révision du décret-loi no $1 / 3$ du 31 août 1992 concernant la création d'un régime de zone franche au Burundi (Art 16). La loi no 1/23 du 24 septembre 2009 relative aux avantages fiscaux prévus dans le code des investissements de 2008 détermine à son tour la nature et la portée desdits avantages.

En son article 16, le code de 2008 prévoit deux régimes de traitement des investissements réalisés sur le territoire burundais. D'une part, il y a le régime général (A) qui peut être considéré comme le régime de droit commun (Code de 2008, Art 7-15). D’autre part, il y a le régime des avantages prévus dans la loi sur la zone franche (B).

\section{Avantages fiscaux du régime général}

La loi du 24 septembre 2009 a apporté des clarifications sur la nature des avantages fiscaux et facilités faisant partie du régime général des investissements (Loi du 24 septembre 2009 art 1). Elle rompt avec l'évocation évasive desdits avantages par le code de 2008 en ses articles 14 et 15 . Cette législation soumet le bénéfice des avantages de ce régime général à la réunion de certaines conditions d'éligibilité.

\section{Conditions d'éligibilité}

La loi du 24 septembre 2009 définit dix objectifs auxquels les entreprises doivent se conformer pour leur éligibilité à ce régime général. L'analyse de ces objectifs permet de conclure qu'ils convergent vers le souci $\mathrm{du}$ législateur d'amener les investissements étrangers à contribuer au développement économique et social du pays d'accueil (Loi du 24 septembre 2009, art 3.1). Ces objectifs ne sont autres qu'une reformulation de la politique du contenu local. Celui-ci comporte des «stipulations, imposed on investors, requiring them to meet certain specified goals with respect to their operations in the host country» (CNUCED, 2003 p. 2). Les dispositions y relatives définissent un paquet de mesures qui exigent aux investisseurs étrangers de contribuer au développement économique. Cette contribution se fait par l'entremise de l'intensification technologique, de la création d'emploi, de la formation d'une main-d'œuvre qualifiée (Loi du 24 septembre 2009, art3.1), de l'élargissement des activités économiques. Le contenu local se reflète aussi dans le recours aux ressources locales dans l'exploitation des activités économiques afin de "remorquer» la compétitivité des entreprises locales (CNUCED, 2003; Nikièma, 2014). L'investissement étranger doit donc impulser l'entrepreneuriat local pour développer un tissu industriel diversifié 
et justement réparti sur le territoire national. En embrassant le contenu local, le code des investissements de 2008 opte pour une orientation économique centrée sur la promotion de l'innovation et la modernisation technologique à l'échelle domestique.

La politique de contenu local est un vieux concept, auquel les pays développés ont recouru à l'étape infantile de leur industrialisation. Les pays en développement se trouvant actuellement dans une phase industriellement juvénile ont adopté cette même politique. Dans une dynamique de divergence d'objectifs des acteurs en présence, cette politique permet de ménager les intérêts des uns et des autres pour faire de l'investissement étranger un rendezvous de gains mutuels pour les partenaires en relations d'affaires. Le recul de l'usage du contenu local dans les politiques d'investissement des pays développés n'est qu'apparent. Ils les ont substituées par d'autres mesures de politique industrielle poursuivant le même but. Il ne s'agit donc pas d'un choix de ne plus chercher à influencer le comportement des entreprises transnationales (Nikièma, 2014 p.4). Jusqu'à une période récente, la Chine utilisait abondamment cette politique de contenu local (Buy Chinese Policy, 2014).

Le contenu local que développe la législation burundaise de 2009 est cependant optionnel. L'investisseur étranger peut choisir de ne pas s'y conformer s'il estime insuffisant le bénéfice de la batterie d'avantages définis en son article 3. En d'autres mots, c'est le degré d'attractivité des incitations accordées qui détermine l'adhésion de l'investisseur au contenu local. Après analyse, nous constatons qu'il y a de l'espace pour relativiser, car les termes de l'article 3 ne laissent pas présager que les objectifs auxquels doit contribuer l'investissement sont cumulatifs et/ou alternatifs. L'appréciation souveraine de l'autorité investie du pouvoir d'évaluation peut donc être rigide ce qui exclurait beaucoup d'investissements pendant que son usage flexible permettrait d'y en admettre beaucoup.

\section{Les avantages proprement dits}

Les avantages qui seront analysés sous cette rubrique concernent les exonérations des droits de mutation et crédit d'impôt ainsi que la réduction du taux d'imposition sur les bénéfices.

\section{Exonération des droits de mutation et crédit d'impôt}

Le premier des avantages fiscaux qu'accorde la loi de 2009 aux investisseurs concerne l'exonération des droits de mutation. Ceux-ci, autrement appelés droit d'enregistrement, sont des taxes perçues par l'État ou par les collectivités locales selon les pays. Le fait générateur de cet «impôt réel» est la vente immobilière, celle-ci entrainant un changement de propriétaire. La liquidation de cet impôt est problématique, car l'évaluation du 
bien telle que conduite actuellement tient compte de la valeur transactionnelle lorsque celle-ci est supérieure à la valeur d'expertise conjointement menée par l'Office burundais des recettes et les services du titre foncier (Ordonnance du 31 juillet 2000).

Aux termes de cette loi de 2009, les entreprises nouvellement créées bénéficient du crédit d'impôt dès la réalisation de leur investissement (Loi du 24 septembre 2009 Art 4.5). Signalons que cet avantage n'existe plus en raison de sa suppression par la loi no 1/38 du 31 décembre 2017 portant fixation du budget général pour l'exercice 2018 (Loi du 31 décembre 2017, Art 75). Cette loi dispose : « le crédit d'impôt prévu à l'article 4 (2) de la loi no $1 / 23$ du 24 septembre 2009 déterminant les avantages fiscaux prévus par la loi no 1/24 du 10 septembre 2008 portant code des investissements du Buruni reste supprimé » (Art 75). Le crédit d'impôt est un assouplissement fiscal permettant de bénéficier d'une réduction de l'impôt par le biais d'un remboursement. L'assujetti doit donc payer et introduire une réclamation en remboursement après exercice. Ce crédit d'impôt est déduit de la valeur d'acquisition des biens investis ainsi que de la base d'amortissement. Le crédit d'impôt s'apparente à une subvention et constitue par conséquent une mesure incitative pour les investissements étrangers. Le crédit d'impôt était un avantage dont la portée financière pouvait encourager les entreprises étrangères à se conformer aux conditions du contenu local. C'est en effet au travers celui-ci que les investisseurs étrangers remplissent quelques objectifs de contribution au développement économique.

La venue de la loi no 1/38 du 31 décembre 2017 portant fixation du budget général pour l'exercice 2018 a vidé la loi de 2009 déterminant les avantages fiscaux de sa raison d'être. Dans la philosophie de son élaboration, le code était présenté comme un outil de promotion des investissements (Code de 2008). En supprimant cet incitatif de crédit d'impôt, le législateur ampute le code des investissements qui devient un instrument désuet et moins attrayant.

Dans un contexte d'une reprise chancelante de l'activité économique qui a subi un coup d'arrêt en raison de la crise politique de 2015, le législateur burundais se devait d'opérer un choix : soit maintenir un crédit d'impôt au rendement d'ailleurs non avéré soit le supprimer pour accroitre ses chances d'optimiser les rentrées fiscales dans les caisses du trésor public. C'est cette deuxième option qui a prévalu.

$\mathrm{Si}$ cette mesure de suppression s'accompagne d'une meilleure administration des investissements étrangers, elle peut permettre de récupérer la quotité de $37 \%$ de crédit d'impôt dont la base de calcul était constituée de biens amortissables investis dans l'entreprise nouvellement créée ainsi qu'aux extensions et réhabilitations d'activités de production de biens et de services (Loi du 24 septembre 2009, Art 4.5). Les actifs immobilisés devaient servir 
dans la société pendant au moins cinq ans (Loi du 24 septembre 2009, Art 4 (5)).

La cession prématurée du bien amortissable engendrait l'obligation pour son bénéficiaire de le rembourser à l'administration fiscale sauf si ladite cession résultait d'une catastrophe naturelle. Dans l'hypothèse d'une cession intervenant en dehors des limites légales, l'assujetti devait payer des intérêts de retard déterminés en fonction des dispositions afférentes au recouvrement d'impôt. Ces intérêts commençaient à courir à partir de la date de l'imputation du crédit au compte du contribuable jusqu'à celle de la cession de l'actif.

\section{Réduction du taux d'imposition sur les bénéfices}

La réduction du taux d'imposition sur les bénéfices est modulée en fonction du nombre d'emplois créés par le développeur du projet d'investissement. Pour un nombre d'emplois se situant entre 50 et 200, l'investisseur bénéficie d'une réduction de $2 \%$. Lorsque le nombre d'emplois créés est supérieur à 200, le taux de réduction sur les bénéfices passe à $5 \%$.

L'importance salariale de l'emploi créée entre en ligne de compte dans la détermination des réductions à octroyer. À ce titre, les emplois qui ouvrent l'accès à cette imposition réduite sont ceux-là dont la rémunération est soumise aux taux d'imposition supérieurs à zéro. L'objectif poursuivi par le législateur est d'écarter l'octroi des avantages aux investisseurs ayant créé des emplois en deçà de la première tranche du revenu soumise au taux zéro d'imposition. Ainsi, pour compter comme emploi donnant accès aux avantages, sa rémunération doit se mesurer à l'aune des taux d'imposition que prévoit la loi sur l'impôt sur le revenu. Cette loi prévoit des tranches d'imposition soumises aux taux progressifs (Loi du 24 janvier 2013, Art 108110). La première tranche du revenu pris en compte va de zéro à cent cinquante mille et son taux d'imposition est de \%. Pour la deuxième tranche du revenu allant de 150001 à 300000 le taux d'imposition est de $20 \%$ et la troisième tranche du revenu qui est supérieur 300000 BIF est soumise à un taux de $30 \%$.

\section{Avantages du régime de zone franche}

Cette section analyse la notion de zone franche et les conditions posées pour accéder aux avantages que la législation sur la zone franche prévoit. L'étude de ce cadre de zone franche débouche sur l'examen des incitatifs fiscaux, douaniers et des avantages en matière d'application de la réglementation du travail par les entreprises franche.

\section{Notion de zone franche}

Dans le but d'insuffler une nouvelle dynamique économique, le Burundi a entamé depuis les années 90 la modernisation de son infrastructure 
législative relative aux investissements. La promotion du climat bienveillant des affaires introduisait la réglementation sur la zone franche (Loi du 31 juillet 2001). Celle-ci désigne le «statut juridique particulier accordé à certaines entreprises établies au Burundi dans les conditions prescrites par la présente loi et les ordonnances ministérielles et au titre duquel elles bénéficient automatiquement d'un ensemble d'exonérations fiscales et douanières ainsi que des mesures d'incitations dans les domaines de la législation du travail, du contrôle des changes, de l'entrée et du séjour des étrangers » (Loi du 31 juillet 2001).

Le Burundi s'est doté d'une première législation sur les zones franches en 1992 à la faveur du décret-loi no 1/3 du 31 août 1992 portant création d'un régime de zone franche au Burundi. Cette législation a été abrogée par la loi no $1 / 015$ du 31 juillet 2001 .

Les dispositions de la loi sur la zone franche déterminent un paquet d'avantages fiscaux, douaniers et sociaux qu'elle concède aux investisseurs nantis du statut d'entreprise de zone franche. Une entreprise de zone franche désigne toute société établie au Burundi selon les règles fixées par les lois en vigueur, à laquelle il a été accordé le régime de zone franche (Loi du 31 juillet 2001, Art 2 (b)). La possession de ce statut se matérialise par l'octroi d'un certificat d'entreprise franche. La zone franche couvre quatre catégories d'entreprises qui sont : entreprises franches agricoles et d'élevage; entreprises franches industrielles et artisanales; entreprises franches commerciales; et entreprises franches de service (Loi du 31 juillet 2001, Art3).

\section{Conditions d'éligibilité à la zone franche}

Sous cette rubrique, nous examinons les conditions communes à toutes les catégories d'entreprises ainsi que les conditions particulières à chacune d'entre elles.

\section{Conditions communes à toutes les catégories d'entreprises}

Pour l'éligibilité au statut de zone franche, toutes les quatre catégories d'entreprises ci-dessus mentionnées doivent se soumettre au respect des règles de l'environnement, de l'hygiène et de la salubrité publique. Pendant que l'imposition de cette condition soulève moins, ou pas, de contestation, le contrôle à sa soumission, provoque des critiques. S'il s'agit d'une déclaration écrite que le promoteur doit produire pour s'engager simplement au respect des prescriptions environnementales, il y a alors une double crainte à son égard.

Soit la condition risque de devenir purement formaliste et par conséquent sans effet escompté sur la protection de l'environnement. Soit la condition est sérieuse et, dans ce cas la déclaration peut être accompagnée d'un plan détaillant les mesures à envisager dans le cadre d'une étude d'impact 
qui anticipe les effets des activités économiques et propose des mesures à prendre pour les relever. Mais cette deuxième hypothèse comporte le danger de décourager les opérateurs économiques en raison des difficultés reliées aux coûts supplémentaires d'acquisition des technologies non polluantes ou des autres mesures environnementales qu'ils pourraient éprouver.

\section{Conditions particulières à chaque entreprise}

Les entreprises de zone franche opérant dans les secteurs agricoles et d'élevage, industriels et artisanaux et de service doivent exporter toute leur production. Une des raisons que nous estimons à la base de ce critère se rapporte au souci d'équilibre de la balance commerciale. Le législateur a voulu encourager la compétitivité commerciale sauf qu'il parait irréaliste de tout exporter. Une partie de la production de ces entreprises trouve sa clientèle sur le marché locale dont il n'est pas inapproprié d'apprivoiser avant de projeter la conquête au-delà des frontières.

Nous estimons que cette politique de zone franche favorise les entreprises étrangères qui ont déjà des moyens de production et une réputation notoire dans leur secteur de production. Or, l'encouragement des investissements étrangers ne devrait pas se faire au détriment des entreprises locales. Cette condition aux allures éliminatoire et discriminatoire nous parait inopportune. Le caractère éliminatoire, qui par ailleurs dépouille de sa raison d'être le statut de zone franche, réside dans le fait que très peu d'entreprises locales à leur démarrage ont la capacité de produire uniquement pour l'exportation. L'industrie naissante du Burundi se trouverait donc en nette défaveur par rapport aux investisseurs étrangers habitués à produire pour exporter. Dans son énoncé actuel, ce critère contribue à rendre aléatoire l'acquisition du statut de zone franche que la loi l'édictant s'était pourtant proposé d'encourager. Le réalisme aurait été de déterminer les quotas d'exportation de la production en tenant compte du facteur temps en termes de délai de vie de l'entreprise et de l'augmentation de la production.

L'autre condition posée seulement aux entreprises relevant de la catégorie des entreprises franches agricoles et d'élevage, industrielle et artisanale concerne la création d'une valeur ajoutée substantielle. La condition est raisonnable, car il faut une création de la richesse qui puisse contrebalancer les avantages que l'État d'accueil consent à ces entreprises. Mais dans le membre de phrase «une valeur ajoutée substantielle», le substantif «substantiel» fait craindre que l'État monnaie cher le statut de zone franche ce qui rendrait illusoire son obtention. L'objectivité des critères pour évaluer le seuil de la valeur ajoutée est aussi un élément déterminant pour "vendre » ou venter les mérites du statut de zone franche aux entreprises de cette catégorie (l'Ordonnance ministérielle fixant le niveau de la valeur ajoutée). 
Nous soutenons qu'il sera contreproductif de miroiter aux investisseurs opérant dans les secteurs agricoles et d'élevage les avantages fiscaux de la zone franche pour les inciter à embrasser la législation, car selon la loi sur l'impôt sur le revenu, le revenu d'affaires provenant d'une activité agricole ou d'élevage est exonéré (Loi du 24 janvier 2013 Art 47 (1)). L'intensité de l'exonération dans la loi sur l'impôt sur le revenu dépasse les avantages fiscaux offerts dans la loi sur la zone franche. Ceci rend moins stimulante cette zone, car en définitive, les charges fiscales est un critère déterminant dans la décision sur la délocalisation et le choix du secteur de l'investissement. Le critère de décision restant concerne les avantages offerts en matière d'application de la législation du travail et du séjour des étrangers au Burundi et nous l'estimons peu déterminant pour influer sur la décision de l'investisseur.

Le législateur a soumis les entreprises franches commerciales aux conditions «d'importation et de réexportation en l'état ou après conditionnement des produits importés dont la liste est fixée par le ministre » (Loi du 31 juillet 2001, Art 4). Cette condition permet certes aux entreprises d'importer. Mais elle exige parallèlement de trouver des débouchés pour réexporter les produits sans ou après leur transformation. Elle encourage certes la création des entreprises de transformation, mais en même temps, cette condition introduit une restriction à l'accès au statut de zone franche. En effet, la législation fiscale burundaise encourage seulement l'exportation qui est imposée à hauteur de zéro pour cent alors que l'importation est lourdement imposée. Dans ces conditions, une entreprise ne trouvera bénéfique de demander le statut que si les coûts à l'importation sont largement inférieurs aux avantages offerts à la réexportation.

Somme toute, ces éléments d'analyse qui portent sur les dispositions pertinentes de cette législation ont mis en exergue les failles qui limitent son efficacité en termes de stimulation des investissements. Les conditions sont restrictives et ceci s'observe par un nombre très restreint d'entreprises ayant recouru à ce statut. Mais il est aussi permis de penser que les investisseurs estiment moins attrayant le régime en raison de son caractère hautement formaliste (Loi du 31 juillet 2001, Art 4 et 32) et surtout de la duplication des avantages fiscaux dans les autres réglementations burundaises beaucoup plus souples (Loi du 24 janvier 2013, Art 47). L'usage limité de ce statut de zone franche découlerait aussi du peu ou pas de publicité qui en est faite par les agences de gestion et d'administration des investissements à l'échelle étatique.

\section{Les incitatifs du régime de zone franche}

La loi sur la zone franche, comme nous l'avons exposé précédemment, octroie des avantages fiscaux et douaniers aux investisseurs étrangers. Elle offre aux investisseurs étrangers un assouplissement dans l'application de 
certaines dispositions en matière de la législation du travail. Et rappelons-le, le code des investissements incorpore en son sein cette législation par l'entremise de l'article 16 du code des investissements (Loi du 31 juillet 2001).

\section{Les incitatifs fiscaux}

Les incitatifs fiscaux dans la loi sur la zone franche reposent sur l'exonération ou la réduction des impôts sur les sociétés, sur le réinvestissement, sur le chiffre d'affaires et sur les dividendes. Le régime de zone franche prévoit aussi des exonérations de taxe de mutation et de droit d'enregistrement sur le montant des augmentations du capital de la société et accorde une réduction du taux d'imposition sur les bénéfices au prorata du nombre d'emplois créés.

Aux termes de la loi de 2013 relative aux impôts sur le revenu, les sociétés établies au Burundi sont assujetties au paiement de l'impôt de $30 \%$ sur les bénéfices des sociétés (Loi du 24 janvier 2013 Art 85 et 94). La loi du 31 juillet 2001 sur la zone franche prévoit 1'exonération totale des impôts sur les bénéfices pendant les 10 premières années d'exploitation pour les entreprises franches. Dès sa onzième année, l'exonération prend fin pour céder la place à une application d'un taux de $15 \%$ qui est réduit lui-même à $10 \%$ lorsque l'entreprise a créé plus de 100 emplois permanents pour les ressortissants burundais (Loi du 31 juillet 2001). En lisant le code général des impôts, le constat est que le législateur y a prévu des exonérations en se référant à celles prévues dans le code des investissements et en reprenant spécifiquement les dispositions de la loi 2002 sur la zone franche en son 12 (Code général des impôts et taxes, livre deux, art 39). La loi relative aux impôts sur le revenu a réitéré également lesdites exonérations en mentionnant que ceux qui sont exonérés en vertu du code des investissements le sont aussi au titre de cette loi relative à l'imposition sur les revenus (Loi du 24 janvier 2013 relative aux impôts sur le revenu Art 87.8).

La loi no $1 / 28$ du 31 décembre 2017 portant fixation du budget général de la République du Burundi pour l'exercice 2018 réaffirme le principe de l'exonération (Loi du 31 décembre 2017 portant fixation du budget général art 24) tout en le réaménageant. Cette législation soumet le bénéfice de l'exonération à la constitution préalable, par le demandeur, d'une caution bancaire de $30 \%$ du montant de l'exonération sollicitée. Cette condition nous parait raisonnable pour décourager les demandes fantaisistes et couvrir les cas de demandes abusives ou les procédures judiciaires.

La même loi énonce que, quel qu'en soit le résultat, l'entreprise reste redevable d'un impôt minimal de $1 \%$ sur le chiffre d'affaires. Cet impôt minimal est d'application lorsque «les bénéfices taxables sont inférieurs au produit obtenu en divisant le chiffre d'affaires par trente (30)». On peut donc 
déduire de cette analyse que l'exonération totale durant les dix premières années prévues dans la loi sur la zone franche est un leurre.

Nous estimons que l'avantage fiscal de réduction de la taxe sur le chiffre d'affaires auquel fait allusion le texte de 2001 sur la zone franche reflète le caractère dépassé de cette réglementation. La taxe sur le chiffre d'affaires était l'ancienne taxe sur les transactions (TT) (Decret-loi du 31 janvier 1989 portant reforme de la taxe sur les transactions et Ordonnance ministérielle no 540/223 du 21/8/1989). Or, celle-ci a été remplacée par la loi no 1/02 du 17 février en 2009 portant institution de la taxe sur la valeur ajoutée «TVA»; elle-même révisée par la loi 1/12 du 29 juillet 2013 (CNUCED, 2010 p. 50).

De plus, la loi budgétaire pour l'exercice 2018 abolit l'exonération de la TVA à l'importation. Dorénavant, la TVA n'est accordée qu'à titre exceptionnel «aux entreprises prioritaires et stratégiques pour l'économie nationale » (Loi no 1/38 du 31 décembre 2017, Art 74 (1)). Cette loi a confié aux ministres ayant les finances et le plan dans leurs attributions le pouvoir de définir «les critères d'éligibilité au statut d'entreprise prioritaire et stratégique ». Nous avons donc un avantage «fantôme» dont la réglementation a rendu caduque.

Dans sa stratégie de rétention des investissements étrangers, cette réglementation accorde aux investisseurs qui réinvestissent $25 \%$ au cours des dix premières années d'exonération totale une réduction de $10 \%$ sur le taux d'imposition réellement applicable. Selon la loi sur l'impôt sur le revenu, le taux d'imposition applicable sur les bénéfices des sociétés est de $30 \%$. L'investisseur paiera donc la différence soit $20 \%$. Le législateur burundais a soumis les investisseurs au paiement de la taxe de $1 \%$ sur le chiffre d'affaires avec possibilité de réduire cette taxe à $0,80 \%$ si l'investisseur a créé plus de 20 emplois permanents pour les ressortissants burundais. Encore une fois, la taxe sur le chiffre d'affaires n'existe plus. Elle a été supplantée par la TVA.

La troisième catégorie d'allégement fiscal est relative à la réduction du taux d'imposition sur les bénéfices en fonction du nombre d'emplois créés par le développeur du projet d'investissement. Pour un nombre d'emplois se situant entre 50 et 200, l'investisseur bénéficie d'une réduction de $2 \%$. Lorsque le nombre d'emplois créés est supérieur à 200, le taux de réduction sur les bénéfices passe à $5 \%$.

\section{Les avantages douaniers}

La loi budgétaire pour l'exercice 2017 semble avoir annulé tous les incitatifs douaniers prévus dans la loi sur la zone franche. En son article 82, cette législation élimine toutes les exonérations de fiscalité indirecte (Loi budgétaire du 31 décembre 2017, Art 82 (1)). La même législation énonce que « toutes les importations exemptées de droits et taxes de douanes prévues par 
les différents instruments juridiques à caractère législatif et réglementaire non repris à l'alinéa précédent sont taxées à $5 \%$ de leur valeur en douanes (à l'exception des fournitures destinées à la présidence de la République et aux forces de la défense nationale et de la police nationale) » (Loi budgétaire du 31 décembre 2017, Art 82 (1)). Ce passage de la loi remet en cause les conditions généreusement fiscales que la loi sur la zone franche avait aménagées au regard de l'implantation et de l'exploitation de l'activité économique d'une entreprise franche. Celle-ci était exonérée de tous droits et taxes directs ou indirects, actuels et futurs sur les matières premières, produits intermédiaires, accessoires et biens d'équipement dont la liste accompagnait le certificat d'entreprise franche (Loi du 31 juillet 2001, Art 18). Désormais cette exonération cède la place à l'application du taux de $5 \%$ de taxe à l'exception des fournitures destinées à la présidence de la République et aux forces de la défense nationale et de la police nationale (Loi budgétaire du 31 décembre 2017, Art 82 (1))).

Nous pensons qu'avec la venue de la loi budgétaire pour l'exercice 2018, les exonérations totales de paiements des taxes indirectes en vigueur ou à créer ainsi que des droits d'enregistrement et de timbre que prévoyaient la loi sur la zone franche sont caduques. La loi sur la zone franche exonérait aussi les investisseurs du paiement de la taxe de transaction, de la taxe de mutation et du droit d'enregistrement sur le montant des augmentations du capital de la société. Elle défiscalisait toutes les opérations d'exportations, c'est-à-dire que les exonérations n'étaient conditionnées qu'aux normes de qualité des produits (Loi du 31 juillet 2001, Art 19). Ce qui, peut-être, reste des avantages douaniers prévus dans la loi sur la zone franche ce sont les facilités d'importer sans licence pour toutes les entreprises franches pour autant que les importations répondent aux normes de qualités en vigueur (Loi du 31 juillet 2001, Art 17).

De la lecture attentive de l'esprit de la loi budgétaire de 2018, nous pouvons conclure que la philosophie dominante est l'imposition. Certains commentaires de cette loi lui reprochent de prendre le contrepied de la politique d'attraction des investissements que prône le gouvernement burundais. Les incitatifs sont des éléments importants dans la promotion des investissements. Mais l'impact immédiat de la suppression des incitatifs par la loi de 2018 doit se mesurer à l'aune du nombre d'entreprises franches qui existent au Burundi. Malheureusement, on peut déplorer l'absence des statistiques sur les entités économiques relevant de la zone franche qui seraient touchées ainsi que la nouvelle charge fiscale sous leur joug.

Dans le contexte «d'étranglement économique » consécutif au retrait des bailleurs de fonds après la crise électorale, le financement du budget national est devenu un enjeu économique de taille. Il fallait opérer un choix et répartir toute la charge fiscale entre les agents économiques internes. La loi de 
2018 doit être comprise comme la volonté de financer le budget national par les recettes internes dont le seul moyen, à court terme, à la portée de l'État est de supprimer les exonérations pour optimiser les rentrées fiscales. Mais avec cette politique fiscale, le Burundi court le danger d'apparaitre comme une destination défavorable pour le peu d'investisseurs étrangers actuel ou potentiel. Si ce risque peut être éloigné par un travail de sensibilisation accrue de l'API pour chercher des investisseurs de nouveaux horizons, il resterait que la comparaison des standards d'exonération dans la sous-région risque d'être un facteur déterminant pour les investisseurs étrangers dans le choix de leur destination.

\section{Les avantages sociaux dans la loi sur la zone franche}

L'allégement des conditions de licenciement des employés et les barèmes salariaux réduits dans sont les quelques exemples d'incitations sociales offertes aux investisseurs et investissements ayant le statut de zone franche. La loi sur la zone franche encourage l'embauche des travailleurs expatriés.

\section{Allégement des conditions de licenciement}

Pour accroitre l'attractivité du Burundi et en faire une destination favorable aux investisseurs étrangers, le code concède quelques avantages relatifs à l'assouplissement en matière d'application de la législation du travail. La loi de 2001 régissant la zone franche, qui fait partie du code, pose le principe du respect de l'application des dispositions générales du code de travail (Loi du 31 juillet 2001, Art 25). Mais aussitôt, cette loi introduit une réserve concernant les articles 26 à 31 de la loi sur la zone franche (Loi du 31 juillet 2001, Art 25). Pareille réserve aménage une prééminence du contenu de ces dispositions par rapport à celles du code de travail portant sur les mêmes matières.

L'article 26 de la loi sur la zone franche constitue un relâchement de la protection de l'employé. Il assouplit les conditions de licenciement et rompt avec la protection que devrait offrir le code de travail si l'application des dispositions subséquentes n'était pas écartée par la réserve. Tel qu'articulé, le texte de l'article 26 permet aux entreprises de zone franche d'allonger la période d'essai. Il institue une durée de 12 mois d'essai pour toutes catégories de travailleurs confondues là où le code de travail en prévoit 6 pour les catégories inférieures avec possibilité d'étendre cette durée à 12 mois pour les catégories cinq et six (Code du travail, Art 31). Ceci consacre un élargissement du droit de licencier. L'entreprise franche peut licencier cet employé «à l'essai» moyennant un préavis d'une semaine ou une compensation équivalente à une semaine de salaire (Loi du 31 juillet 2001, Art 26.a). 
La réglementation sur la zone franche est silencieuse quant au paiement des dommages-intérêts pour rupture abusive. Elle n'évoque pas non plus les indemnités de brusque rupture de contrat de travail. Cet assouplissement, synonyme de recul des droits sociaux des employés, permet à l'entreprise franche de "se débarrasser » des employés à un coût réduit.

Une entreprise de zone franche peut, après la période d'essai, licencier le travailleur «conformément aux termes de son contrat de travail avec l'employeur (Loi du 31 juillet 2001, Art 26 (b)). Cependant, l'employeur sera tenu de lui donner un préavis de deux semaines, ou de lui verser une compensation équivalente. Dans les deux cas, l'employeur devra également verser une indemnisation d'un montant équivalent à deux semaines de salaire par année de service du travailleur (Loi du 31 juillet 2001, Art 26 (b)). Au regard de cette disposition, l'employée est à la merci de son employeur que l'on soit dans le cas du contrat à durée déterminée ou indéterminée.

En effet, le caractère adhésif fait du contrat de travail, aussi bien dans sa négociation que dans son application, un «empire» où prédomine le pouvoir économique de l'employeur. En abandonnant les conditions de licenciement à la seule appréciation des clauses du contrat, et ceci par l'employeur lui-même, ce dernier risque de se comporter comme « un lion dans la basse cour»; ce qui reviendrait à lui conférer un pouvoir discrétionnaire de licenciement.

Lorsque les parties ont pour base juridique un contrat à durée indéterminée, le code de travail reconnait un droit réciproque de se séparer de l'autre à tout moment sous réserve de respecter les ses dispositions (Code du travail, Art.47). La loi sur la zone franche a semblé mettre en avant le principe de la liberté contractuelle qu'elle a aussitôt diluée en pensant l'avoir encadrée.

Le manque d'encadrement légal de la rupture constitue une des grandes lacunes de la loi sur la zone franche. En dehors de l'hypothèse de faute lourde, l'application de l'article 26 de la loi sur la zone franche est en déphasage avec le code de travail burundais qui soumet au consentement mutuel la rupture prématurée du contrat à durée déterminée (Code du travail, Art.46). Il s'agit encore une fois d'une entorse à la réglementation du travail qui fragilise davantage l'accès à l'emploi et nivelle par le bas les conditions de travail des employés locaux. Aux termes de cette disposition, le coût du licenciement d'un employé de zone franche ne peut dépasser un mois de salaire pour une année de service. Relativement à ce coût, la loi sur la zone franche dilue les conséquences pécuniaires d'un licenciement abusif opéré par l'employeur. L'esprit du code de travail est tout autre car, le licenciement après une année de service est dispendieux surtout lorsqu'il est abusif (Code du travail, Art.60). 


\section{Barèmes salariaux réduits}

Le salaire et la durée du travail sont négociés librement sous cette législation de zone franche. Cela n'a rien d'illégal en soi. Mais, lorsque cette loi ajoute que «cependant le salaire ne peut être inférieur au salaire interprofessionnel minimum fixé par la loi » (Loi du 31 juillet 2001, Art 27), l'investisseur étranger reçoit un chèque en blanc pour sou payer les travailleurs surtout ceux ayant un niveau d'instruction élevée. Nous en déduisons que selon cette disposition de l'article 27, toute rémunération qui n'est pas en deçà du minimum est légale. Or, la définition de salaire minimum aurait du prendre en considération les critères de catégories et de niveau d'étude. Les rédacteurs de la loi sur la zone franche ont perdu de vue que les salaires minimums au Burundi sont régis par une réglementation archaïque, en déphasage avec les réalités socio- économiques actuelles.

L'ordonnance fixant les salaires minimums date de 1988 (Ordonnance du 30 avril 1988, art 2). Elle fixe un salaire minimum à 160 BIF par jour dans les Centres urbains de Gitega et de Bujumbura et 105 BIF par jour dans les zones rurales. La dégradation des conditions de vie consécutive à l'environnement économique et sociale a poussé à la hausse ces montants qui jusqu'à présent n'ont pas encore subi la censure légale. En milieu rural le salaire oscille entre 2000 et 1500 et en milieu urbain, il se situe entre 3500 et 5000. Ces taux ne concernent que les catégories de travailleurs sans aucune qualification professionnelle ou intellectuelle. Si c'est l'application alors de cette ordonnance de 1988 que le code de travail recommande, il est de réalité notoire que ces taux ne sont appliqués nulle part au Burundi et que le gouvernement ferait mieux de suivre la pratique tout en l'améliorant.

Relativement à la rémunération des heures supplémentaires, la loi sur la zone franche rémunère les heures supplémentaires à raison de 1,5 fois le salaire de base pendant les dix premières heures. Mais, le code fixe ce taux à $35 \%$ chacune des deux premières heures effectuées au-delà de la durée légale hebdomadaire du travail ou de la durée hebdomadaire de présence (Ordonnance du 9 mai 1979). Dans les entreprises où le code de travail est d'application, toute heure dépassant les deux premières est rémunérée à hauteur de soixante pour cent pour chacune des heures suivantes (Ordonnance du 9 mai 1979) tandis que dans la zone franche, c'est après dix heures supplémentaires que les cinq heures suivant sont rémunérées à hauteur de deux fois le salaire de base. Dans le code du travail, ce pourcentage est calculé sur la base de la rémunération horaire brute de l'employé. En guise de synthèse, dans la législation sur la zone franche, tout se passe comme si la rémunération des heures supplémentaires est plafonnée à quinze heures. 


\section{Assouplissement des conditions d'embauche des travailleurs expatriés}

Les investisseurs étrangers disposent du droit d'embaucher des cadres supérieurs spécialisés étrangers ayant un permis de travail sans imposer de limitation de nombre. Le renouvellement du permis se fait par tacite reconduction. Cette autorisation pose la question de la qualité d'emploi réservée à la main-d'œuvre locale. Certes, c'est un avantage pour les investisseurs étrangers. Mais, il n'est pas en arrimage avec le contenu local que prône l'ordonnance de 2009 sur la détermination des avantages qu'offre le code des investissements en ses articles 14 et 15 . Une partie des retombées de l'investissement étranger consistent au transfert de technologie, de création d'emploi, et de compétence de gestion. L'article 30 est à l'antipode du but poursuivi par le code de 2008. En effet, le législateur burundais a offert des incitatifs dans la perspective d'en tirer les gains. Or, le texte de l'article 30 diminue la possibilité d'embaucher les cadres burundais, et répond moins, ou pas du tout, à la réalité actuelle du marché de travail. Les compétences ont augmenté et se sont diversifiées, rendant moins nécessaire le recours aux travailleurs expatriés. En intégrant cette législation dans le code de 2008, le législateur a introduit une incohérence qui hypothèque les avantages qu'il escomptait tirés de la présence des investisseurs étrangers dans la zone franche.

\section{Conclusion}

L'attraction des investissements étrangers a toujours été, et le restera pour longtemps, un élément central de la politique de développement économique des pays aussi bien pauvres que riches. De l'hostilité à la méfiance des années d'après les indépendances s'est substituée une confiance, parfois délibérément fanatique, pour apprivoiser un climat serein pour les investissements et les investisseurs étrangers. Pour le Burundi, une des manifestations de cette hospitalité est l'inclusion graduelle dans les diverses générations des codes d'investissements des dispositions substantielles protégeant les investissements. Ceux-ci sont devenus des sources de flux financiers sur lesquelles le Burundi doit capitaliser pour rompre la culture de dépendance envers la métropole.

La compétitivité entre les pays demandeurs de capitaux étrangers justifie l'attitude confessionnelle que le législateur burundais a adoptée dans la rédaction du code des investissements de 2008. Cette législation définit un paquet d'avantages douaniers et fiscaux à l'endroit des investissements et des investisseurs étrangers. Par ailleurs, elle comporte des incitatifs sociaux sous forme d'assouplissements en matière d'application de la réglementation du travail dans les entreprises ayant le statut d'entreprises de zone franche.

Cette réflexion a procédé à l'évaluation du traitement des investisseurs étrangers dans le code de 2008 sur les incitatifs fiscaux, douaniers et sociaux. 
Elle a démontré que les lois budgétaires pour l'exercice 2017 et 2018 ont opéré un grignotage sur ces avantages d'ordre fiscal que contient le code. La conclusion que l'on dégage de cette incursion des lois fiscales dans l'empire du code est que lesdits avantages sont plus théoriques que réels. Certains de ces avantages ont même été supprimés.

Les concessions en matière de législation du travail ont subi l'usure du temps et sont en décalage avec certaines réalités socio-économiques du pays. En concluant que cet arsenal juridique relatif aux investissements étrangers est vétuste, notre modeste contribution consiste à faire un clin d'œil au législateur burundais d'accélérer les procédures de révisions du code de 2008 et de s'engager dans la logique de rajeunissement des autres réglementations nationales relatives aux investissements.

La politique d'attraction des investissements et le vœu renouvelé de rompre avec la dépendance économique exigent du législateur burundais de renforcer la convergence, la prévisibilité et la transparence dans la détermination et l'octroi des avantages afférant aux incitatifs fiscaux et douaniers. Cette dynamique nécessite de faire un travail de compilation de ces incitatifs pour en détecter ceux qui sont devenus archaïques et procéder à leur nouvelle redéfinition pour en effectuer une nouvelle codification.

\section{References:}

1. Agence égyptienne GAFI, en ligne: Gafi <http://www.gafinet.org/English/Pages/ GAFIMission.aspx>.

2. CNUCED (2010). Différends entre investisseurs et État : prévention et modes de règlement autres que l'arbitrage. New York; Genève, Collection Études de la CNUCED sur les politiques d'investissement international au service du développement : Nations Unies.

3. CNUCED (2010). Examen de la politique d'investissement Burundi. New York; Genève : Nations Unies.

4. CNUCED, (2003). FDI and Performance Requirements: New Evidence from Selected Countries, Genève : Nations Unies.

5. Decret-loi no 1/04 du 31/1/1989 portant réforme de la taxe sur les transactions (Burundi).

6. Décret-loi no 1/8 du 4/4/1979 portant Code des investissements du Burundi, B.O.B. no 6/79, pp.261-267.

7. . Décret-loi no $1 / 82$ du 25/8/1967 portant Code des investissements $d u$ Burundi, B.O.B. no 9/67, pp.338-343.

8. Décret-loi no 11/03 du 07/7 1993 portant Révision du Code du travail du Burundi (B.O.B., 1993, no 9, p. 453).

9. Hatem, F. (1993). Les politiques d'attractivité en Europe de l'Ouest : Délégation aux investissements internationaux, Paris. 
10. Houben c. la République du Burundi (ICSID Case No. ARB/13/7), sentence du 12 janvier 2016.

11. Laviec, J P. (2015). Protection et promotion des investissements : Étude de droit international économique: Graduate Institute Publications.

12. Loi du 6/8/1963 portant institution d'un Code des investissements $d u$ Burundi, B.O.B. no 12 bis/1963, p.416.

13. Loi no 1/005 du 14/1/1987 portant Code des investissements $d u$ Burundi B.O.B. 1987, no 6/87, pp.193-198.

14. Loi no $1 / 015 \mathrm{du} 31 / 7 / 2001$ portant révision du décret-loi no $1 / 3 \mathrm{du}$ 31/8/1992 portant création d'un régime de zone franche au Burundi, B.O.B. no $7 /$ bis 2001 , pp.794-801..

15. Loi no 1/24 du 10/9/2008 portant code des investissements du Burundi.

16. Loi no 1/23 du 24/9/2009 déterminant les avantages fiscaux prévus par la loi no 1/24 du 10/9/2008 portant code des investissements du Burundi.

17. Loi no 1/02 du 24 janvier 2013 relative aux impôts sur les revenus (Burundi).

18. Loi 1/12 du 29/7/2013 ayant révisé celle no 1/02 du 17 février en 2009 portant institution de la taxe sur la valeur ajoutée "TVA» (Burundi).

19. Loi no 1/20 du 31 décembre 2016 portant Fixation du Budget Général de la République du Burundi pour l'exercice 2017.

20. Loi no $1 / 38$ de la 31/7/2017 portant fixation du budget général de la République du Burundi pour l'exercice 2018.

21. Morisset, J. \& Kelly, A.J. (2004). The Effectiveness of Promotion Agencies at Attracting Foreign Direct Investment, Washington, DC: World Bank.

22. Nikièma, S.H. (2014). Performance Requirements in Investment Treaties Best Practices, Published by the International Institute for Sustainable Development.

23. Ordonnance ministérielle du 31/7/2000 portant fixation de la base de la taxe sur les transactions pour les ventes immobilières (Burundi).

24. Ordonnance ministérielle no 540/223 des 21/8/1989 portant modalités d'exécution du décret-loi no 1/04 du 31/1/1989 portant reforme de la taxe sur les transactions (Burundi).

25. Ordonnance ministérielle no 630/116, du 9/5/1979 portant Fixation des taux minima de majoration des heures supplémentaires, des heures effectuées de nuit, le jour de repos hebdomadaire et les jours fériés (B.O.B., 1979, no 7, p. 336) (Burundi)..

26. Ordonnance ministérielle no 650/11/88 du 30/4/1988 portant Fixation du salaire minimum interprofessionnel garanti (SMIG) dans le secteur structurel de l'économie nationale (Burundi). 
27. Republic of China. Buy Chinese policy directives issued by the Chinese government in April 2009. 